\title{
Effect of Storage at Temperature (4C) on Complete Blood Count Parameters
}

\author{
Naif Taleb Ali \\ Department of Haematology, Faculty of Medical Laboratory Sciences, Al neelin University, Khartoum, Sudan \\ Email address: \\ Naiftaleb@yahoo.com, Naifalhag@gmail.com \\ To cite this article: \\ Naif Taleb Ali. Effect of Storage at Temperature (4C) on Complete Blood Count Parameters. Journal of Cancer Treatment and Research. \\ Vol. 5, No. 2, 2017, pp. 7-10. doi: 10.11648/j.jctr.20170502.11
}

Received: November 8, 2016; Accepted: March 11, 2017; Published: March 17, 2017

\begin{abstract}
Background: Complete blood counts are done to monitor overall health, to screen for some diseases, to confirm a diagnosis of some medical conditions, to monitor a medical condition, and to monitor changes in the body caused by medical treatments. Most blood counts today include a CBC count and leukocyte differential count (LDC) (that is, not just the total WBC count but also the count of each WBC type, such as neutrophils, eosinophils, basophils, monocytes, and lymphocytes). More sophisticated modern analyzers can provide extended differential counts. Objective: This study aimed to assess the effect of Storage and Temperature (4C) on Complete Blood Count Parameters. Materials and Methods: This were cross sectional study conducted in Khartoum state in 2016. It included 100 samples, complete blood count conducted to all 100 samples freshly, after 24hours, 48 hours and 72 hours. Mindary BC-2800 hematological analyzer was used to estimate CBC. Results: There was a significant difference in Hematocrit, mean cell volume, MCHC, red cell distribution width, Lymphocytes, granulocyte \%, and platelets indices (P less than 0.05). Lymphocytes count, Mixed count, Lymphocytes \%, Mixed \% and PCT did not show statistically difference ant it were insignificant with $\mathrm{P}$. values $(\mathrm{P}=0.067),(\mathrm{P}=0.080),(\mathrm{P}=0.072),(\mathrm{P}=0.088)$ and $(\mathrm{P}=0.060)$ respectively. Conclusion: This study concluded that storage caused significant difference in Hematocrit, mean cell volume, MCHC, red cell distribution width, Lymphocytes, granulocyte \%, and platelets indices (P less than 0.05 ).
\end{abstract}

Keywords: Complete Blood Count, Lymphocytes, WBCs

\section{Introduction}

A complete blood count (CBC), also known as a full blood count (FBC), is a blood panel requested by a doctor or other medical professional that gives information about the cells in a patient's blood, such as the cell count for each cell type and the concentrations of various proteins and minerals. A scientist or lab technician performs the requested testing and provides the requesting medical professional with the results of the CBC. [1] Complete blood counts are done to monitor overall health, to screen for some diseases, to confirm a diagnosis of some medical conditions, to monitor a medical condition, and to monitor changes in the body caused by medical treatments. [2]

For patients who need blood transfusion, a blood count may be used to get data which would help plan an amount of treatment. [3] In such cases, the person should have only one blood count for the day, and the transfusion of red blood cells or platelets should be planned based on that. [3] Multiple blood draws and counts throughout the day are an excessive use of phlebotomy and can lead to unnecessary additional transfusions, and the extra unnecessary treatment would be outside of medical guidelines. [3] Most blood counts today include a CBC count and leukocyte differential count (LDC) (that is, not just the total WBC count but also the count of each WBC type, such as neutrophils, eosinophils, basophils, monocytes, and lymphocytes). More sophisticated modern analyzers can provide extended differential counts, which include hematopoietic progenitor cells, immature granulocytes, and erythroblasts. [4] The blood is well mixed (though not shaken) and placed on a rack in the analyzer. This instrument has flow cells, photometers and apertures that analyze different elements in the blood. The cell counting component counts the numbers and types of different cells within the blood. The results are printed out or sent to a computer for review. [4] In general, hematology 
testing is performed on EDTA- (lavender top tube) anticoagulated blood. This is the only type of anticoagulant that can be assayed with our hematology analyzer, therefore all hematology tests performed with this analyzer (routine hemograms, red and white cell counts, etc) will only be done from EDTA tubes. [5] Haematological assay $\mathrm{CBC}$ which contained the follow parameters; WBCs, RBCs, HGB, HCT, MCV, MCH, MCHC, PLT, Lymp \%, MXD \%, NEUT \%, Lymp\#, MXD\#, NEUT\#, RDWsd, RDWcv, PDW, MPV and P-LCR which have been investigated by using Sysmex ${ }^{\circledR} \mathrm{Kx}$ $21 \mathrm{~N}$ hematological analyzer.

Each CBC parameters were counted using the direct current detection method with coincidence correction. Automatic discriminators separate the cell populations based on complex algorithms. The intensity of the electronic pulse from each analyzed cell is proportional to the cell volume. The hematocrit (HCT) is directly determined based on the red cell count and volume detection ofeach individual RBC. Even with samples at extremely low or unusually high concentrations. Data analysis

All statistical analyses were performed by SPSS software version (20). Continuous variables were expressed as mean and standard deviation. In the analytical phase, One way Annova was applied to compare means and make Comparison between groups. Correlations was determined and $\mathrm{p}$-value $<0.05$ was considered significant. Haematological assay $\mathrm{CBC}$ which contained the follow parameters; WBCs, RBCs, HGB, HCT, MCV, MCH, MCHC, PLT, Lymp \%, MXD \%, NEUT \%, Lymp\#, MXD\#, NEUT\#, RDWsd, RDWcv, PDW, MPV and P-LCR which have been investigated by using Sysmex ${ }^{\circledR} \mathrm{Kx}-21 \mathrm{~N}$ hematological analyzer.

Each CBC parameters were counted using the direct current detection method with coincidence correction. Automatic discriminators separate the cell populations based on complex algorithms. The intensity of the electronic pulse from each analyzed cell is proportional to the cell volume. The hematocrit (HCT) is directly determined based on the red cell count and volume detection ofeach individual RBC. Even with samples at extremely low or unusually high concentrations. Data analysis

All statistical analyses were performed by SPSS software version (20). Continuous variables were expressed as mean and standard deviation. In the analytical phase, One way Annova was applied to compare means and make Comparison between groups. Correlations was determined and $\mathrm{p}$-value $<0.05$ was considered significant.

\section{Materials and Methods}

This study is cross sectional study, conducted in Khartoum state, Sudan, in the period from August to October 2016. It is included 100 participants.

Blood samples were collected from all subjects in K3EDTA containers for measurement of complete blood count using Mindray ${ }^{\circledR}$ BC-2800 hematological analyzer. The volanteers consisted of healthy volunteers without a medical history of diseases. This study was approved by ethical committee of the faculty of medical laboratory sciences, Alneelin University, and informed consent was obtained from each participant before sample collection.

\section{Haematological Assay}

WBCs, RBCs and PLTs were counted using the direct current detection method with coincidence correction. Automatic discriminators separate the cell populations based on complex algorithms. The intensity of the electronic pulse from each analyzed cell is proportional to the cell volume. The hematocrit (HCT) is directly determined based on the red cell count and volume detection of each individual RBC. Even with samples at extremely low or unusually high concentrations, the Mindary cell counters analyze WBCs, RBCs and PLTs with uncompromised precision and accuracy.

\section{Results}

In total of 100 samples collected in this study, Complete Blood Count was estimated for all samples using Mindary BC-2800 hematological analyzer. The CBC was done on fresh sample, after 24 hour, after 48 hours and after 72 hours. The results found that the hemoglobin (mean+/-SD) on fresh sample, 24 hours, 48 hours and 72 hours was $11.4(+/-1.90)$, $11.41(+/-1.93), 11.45(+/-1.90$ and $11.24(+/-1.92)$ respectively. There was no statistically significant difference in hemoglobin among different periods $(\mathrm{P}=0.998)$. The red blood cells (mean+/-SD) on fresh sample, 24 hours, 48 hours and 72 hours were $4.54(+/-0.54), 4.55(+/-0.22), 4.60(+/-$ $0.43)$ and $4.52(+/-0.87)$ respectively. The difference between periods on red blood cell was in significant $(\mathrm{P}=0.976)$. Hematocrit (mean $+/-\mathrm{SD})$ on fresh sample, 24 hours, 48 hours and 72 hours were $38.9(+/-3.1), 40.1(+/-$ $5.32), 42.9(+/-5.34)$ and $44.0(+/-4.23)$ respectively. There is strong statistically significant difference on hematocrit results $(\mathrm{P}=0.000)$, there was increase in hematocrit with time. Mean cell volume (mean+/- SD) on fresh sample, 24 hours, 48 hours and 72 hours were $77.10(+/-1.9), 78.80(+/-1.65)$, $83.30(+/-2.87)$ and $84.00(+/-1.02)$ respectively, there was strongly significant increase difference in $\mathrm{MCV}(\mathrm{P}=0.000)$. Mean cell hemoglobin (mean+/-SD) on fresh sample, 24 hours, 48 hours and 72 hours were $26.25(+/-1.65), 26.80(+/-$ $1.01), 26.76(+/-2.27)$ and $26.7(+/-1.54)$ respectively, there was no significant difference in mean cell hemoglobin $(\mathrm{P}=0.900)$. Mean cell hemoglobin concentration (mean+/SD) on fresh sample, 24 hours, 48 hours and 72 hours were $34.00(+/-3.27), 31.60(+/-2.34), 29.00(+/-3.01)$ and 28.00 $(+/-2.98)$ respectively, there was significant difference in mean cell hemoglobin concentration $(\mathrm{P}=0.01)$ with decrease in concentration with time.

Red cell distribution width (mean+/-SD) on fresh sample, 24 hours, 48 hours and 72 hours were $14.30(+/-1.05), 14.80$ $(+/-1.17), 15.30(+/-0.98)$ and $15.70(+/-2.13)$ respectively, there was significant difference in $\operatorname{RDW}(\mathrm{P}=0.034)$ with increase with time. Total white blood cells (mean+/-SD) on 
fresh sample, 24 hours, 48 hours and 72 hours were $7.44(+/-$ 4.7), $7.47(+/-3.33), 7.65(+/-2.88)$ and $6.47(+/-2.50)$ respectively, there was no significant difference in TWBCs $(\mathrm{P}=0.193)$. Granulocytes \% (mean+/-SD) on fresh sample, 24 hours, 48 hours and 72 hours were $56.71(+/-1.56), 50.69(+/-$ 1.87), $32.57(+/-1.65)$ and $30.63(+/-1.09)$ respectively, there was strongly significant difference in Granulocytes \% $(\mathrm{P}=0.000)$ with decrease with time. Platelets (mean+/-SD) on fresh sample, 24 hours, 48 hours and 72 hours were 250.79 (+/-33.76), $273.85(+/-14.21), 246.79(+/-25.61)$ and 242.71 $(+/-14.87)$ respectively, there was strongly significant difference in Platelets $(\mathrm{P}=0.000)$ and there was decrease with time. Platelet indices showed difference in mean platelet volume (mean+/-SD) on fresh sample, 24 hours, 48 hours and 72 hours were $9.30(+/-1.78), 9.70(+/-1.64), 10.60(+/-$ $1.017)$ and $10.90(+/-1.92)$ respectively, there was strongly significant difference in Platelets $(\mathrm{P}=0.032)$ and there was increase with time. Platelet distribution width also showed difference in (mean+/-SD) on fresh sample, 24 hours, 48 hours and 72 hours were $14.70(+/-3.84), 14.80(+/-3.54)$, $15.30(+/-2.59)$ and $15.60(+/-4.81)$ respectively, there was strongly significant difference in Platelets $(\mathrm{P}=0.022)$ and there was increase with time. mean platelet volume (mean $+/$ SD) on fresh sample, 24 hours, 48 hours and 72 hours were $9.30(+/-1.78), 9.70(+/-1.64), 10.60(+/-1.017)$ and $10.90(+/-$ 1.92) respectively, there was strongly significant difference in MPV $(\mathrm{P}=0.032)$ and there was increase with time Lymphocytes count and Lymphocytes \% showed statistically significant difference $(\mathrm{P}=0.04)$ and $(\mathrm{P}=0.025)$. Mixed count, Mixed \% and PCT did not show statistically difference ant it were insignificant with $\mathrm{P}$. values $(\mathrm{P}=0.080),(\mathrm{P}=0.088)$ and $(\mathrm{P}=0.060)$ respectively.

Table 1. Show the mean and STD of CBC parameters and relation with time.

\begin{tabular}{|c|c|c|c|c|c|}
\hline & Fresh (mean+/-SD) & 24 hour & 48 hour & 72 hour & p. value \\
\hline Haemoglobin (g/dl) & $11.4(+/-1.90)$ & $11.41(+/-1.93)$ & $11.45(+/-1.90)$ & $11.24(+/-1.92)$ & 0.998 \\
\hline $\mathrm{RBCs}$ & $4.54(+/-0.54)$ & $4.55(+/-0.22)$ & $4.60(+/-0.43)$ & $4.52(+/-0.87)$ & 0.976 \\
\hline Hct & $38.9(+/-3.1)$ & $40.1(+/-5.32)$ & $42.9(+/-5.34)$ & $44.0(+/-4.23)$ & 0.000 \\
\hline MCV & $77.10(+/-1.9)$ & $78.80(+/-1.65)$ & $83.30(+/-2.87)$ & $84.00(+/-1.02)$ & 0.000 \\
\hline $\mathrm{MCH}$ & $26.25(+/-1.65)$ & $26.80(+/-1.01)$ & $26.76(+/-2.27)$ & $26.7(+/-1.54)$ & 0.900 \\
\hline $\mathrm{MCHC}$ & $34.00(+/-3.27)$ & $31.60(+/-2.34)$ & $29.00(+/-3.01)$ & $28.00(+/-2.98)$ & 0.01 \\
\hline RDW-C & $14.30(+/-1.05)$ & $14.80(+/-1.17)$ & $15.30(+/-0.98)$ & $15.70(+/-2.13)$ & 0.034 \\
\hline RDW-S & $47.30(+/-1.3)$ & $47.80(+/-1.03)$ & $46.90(+/-1.99)$ & $47.0(+/-1.56)$ & 0.767 \\
\hline TWBCs & $7.44(+/-4.7)$ & $7.47(+/-3.33)$ & $7.65(+/-2.88)$ & $6.47(+/-2.50)$ & 0.193 \\
\hline Lymph \% & $33.57(+/-0.34)$ & $34.67(+/-0.65)$ & $48.64(+/-0.12)$ & $48.69(+/-0.32)$ & 0.025 \\
\hline MXD \% & $8.98(+/-0.03)$ & $14.06(+/-0.43)$ & $19.21(+/-0.021)$ & $18.78(+/-0.011)$ & 0.030 \\
\hline Granul \% & $56.71(+/-1.56)$ & $50.69(+/-1.87)$ & $32.57(+/-1.65)$ & $30.63(+/-1.09)$ & 0.000 \\
\hline Lymph\# & $2.80(0.34)$ & $2.90(+/-0.65)$ & $3.50(+/-0.12)$ & $3.80(+/-0.32)$ & 0.04 \\
\hline MXD\# & $1.40(+/-0.03)$ & $1.80(+/-0.43)$ & $2.6(+/-0.021)$ & $1.90(+/-0.011)$ & 0.02 \\
\hline Granul\# & $3.90(+/-1.56)$ & $3.50(+/-1.87)$ & $2.90(+/-1.65)$ & $2.92(+/-1.09)$ & 0.001 \\
\hline PLTs & $250.79(+/-33.76)$ & $273.85(+/-14.21)$ & $246.79(+/-25.61)$ & $242.71(+/-14.87)$ & 0.000 \\
\hline MPV & $9.30(+/-1.78)$ & $9.70(+/-1.64)$ & $10.60(+/-1.017)$ & $10.90(+/-1.92)$ & 0.032 \\
\hline PDW & $14.70(+/-3.84)$ & $14.80(+/-3.54)$ & $15.30(+/-2.59)$ & $15.60(+/-4.81)$ & 0.022 \\
\hline \multirow[t]{2}{*}{ PCT } & $.35(+/-0.032)$ & $0.46(+/-0.019)$ & $0.45(+/-0.019)$ & $0.49(+/-0.078)$ & 0.060 \\
\hline & Fresh $($ mean+/-SD) & 24 hour & 48 hour & 72 hour & p. value \\
\hline
\end{tabular}

\section{Discussion}

There is substantial evidence from in vitro studies documenting the change that haematological parameters undergo during storage. It was observed that heamatol- ogical parameters analyze at $4^{\circ} \mathrm{C}$ increase and decreased.

Our study show agreement with study done in Turkey 2011 by Turan Turhan et al. [6] Their results found that Refrigerated storage caused a decline in WBC and platelet counts. MPV was increased in the specimens whereas MCHC was decreased. This study show agreement with our study in White blood cells, mean platelet volume and mean cell hemoglobin concentration.

Another study was done in USA on 2015 by Brent L. Wood, et al. [7] the study aimed to study effect of storage at $4 \mathrm{C}$ on complete blood count. The results were that three directly measured parameters, $\mathrm{RBC}$, hemoglobin, and platelet values, were essentially unchanged after storage at $4^{\circ} \mathrm{C}$. In contrast, while a statistically significant decrease $(P<.0008)$ in the WBC count was observed with storage at $4^{\circ} \mathrm{C}$. This study also show agreement with our study.

The difference between our results and other results may be due environmental factors, different instruments use or technical difference.

\section{Conclusion}

This study concluded that storage caused significant difference in Hematocrit, mean cell volume, MCHC, red cell distribution width, Lymphocytes, granulocyte \%, and platelets indices (P less than 0.05). Data analysis

All statistical analyses were performed by SPSS software version (20). Continuous variables were expressed as mean and standard deviation. In the analytical phase, One way Annova was applied to compare means and make Comparison between groups. Correlations was determined and $\mathrm{p}$-value $<0.05$ was considered significant. 


\section{References}

[1] Verso, ML (May 1962). "The Evolution of Blood Counting Techniques" (PDF). Read at a meeting of the Section of the History of Medicine, First Australian Medical Congress. 8: $149-58$.

[2] Mayo Clinic (14 February 2014). "Complete blood count (CBC) Why it's done - Tests and Procedures". mayoclinic. org. Retrieved 29 July 2014.

[3] American Association of Blood Banks (24 April 2014), "Five Things Physicians and Patients Should Question", Choosing Wisely: an initiative of the ABIM Foundation, American Association of Blood Banks, retrieved 25 July 2014.

[4] Buttarello, M; Plebani, M (Jul 2008). "Automated blood cell counts: state of the art.". American journal of clinical pathology. 130 (1): 104-16.

[5] National Committee for Clinical Laboratory Standards. Tubes and additives for venous blood specimen collection; approved standard - fifth edition. Document H1-A5. Wayne, PA: NCCLS, 2003.

[6] National Committee for Clinical Laboratory Standards. Tubes and additives for venous blood specimen collection; approved standard - fifth edition. Document H1-A5. Wayne, PA: NCCLS, 2003.

[7] Registered: 10 June 2010; Accepted: 2 May 2011 [Kayı1 Tarihi: 10 Haziran 2010; Kabul Tarihi: 02 Mayss 2011].

[8] Kjeldsberg CR, Hershgold EJ. Spurious thrombocytopenia. JAMA. 1974; 227 (6): 628-30.

[9] Evans V. Platelet morphology and the blood smear. J Med Technol. 1984; 1: 689-95.

[10] Shreiner DP, Bell WR. Psueudothrombocytopenia: Manifestation of a new type of platelet agglutinin. Blood. 1973; 42: 541-49. 